\title{
Proximity within Interphase Chromosome Contributes to the Breakpoint Distribution in Radiation-induced Intrachromosomal Exchanges
}

\author{
YeZhang ${ }^{1,2}$, JimmyUhlemeyer ${ }^{3}$, Megumi Hada ${ }^{1,2}$, A.Asaithamby ${ }^{4}$, David J.Chen ${ }^{4}$, HongluWu ${ }^{1}$ \\ ${ }^{1}$ NASA Johnson Space Center, Houston, Texas, ${ }^{2}$ Wyle Laboratories, Houston, TX,
}

${ }^{3}$ Texas A\&M University, College Station, Texas, ${ }^{4}$ University of Texas Southwestern Medical Center, Texas

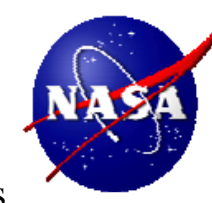

\section{BACKGROUND}

Previously, we reported that breaks involved in chromosome aberrations were clustered in several regions of chromosome3 in human mammary epithelial cells after exposures to either low-or high-LET radiation. In particular, breaks in certain regions of the chromosome tended to rejoin with each other to form an intrachromosome exchange event. This study tests the hypothesis that proximity within a single chromosome in interphase cell nuclei contributes to the distribution of radiation-induced chromosome breaks. Chromosome 3 in G1 human mammary epithelial cells was hybridized with the multicolor banding in situ hybridization (mBAND) probes that distinguish the chromosome in six differently colored regions, and the location of these regions was measured with a laser confocal microscope. Results of the study indicated that, on a multi-mega base pair scale of the DNA, the arrangement of chromatin was non-random. Both telomere regions tended to be located towards the exterior of the chromosome domain, whereas the centromere region towards the interior. In addition, the interior of the chromosome domain was preferentially occupied by the p-arm of the chromatin, which is consistent with our previous finding of intrachromosome exchanges involving breaks on the p-arm and in the centromere region of chromosome3. Other factors, such as the fragile sites in the 3p21 band and gene regulation, may also contribute to the breakpoint distribution in radiation-induced chromosome aberrations. Further investigations suggest that the 3D chromosome folding is cell type and culture condition dependent.

\section{MATERIALS AND METHODS}

Human mammary epithelial cells (CH184B5F5/M10) were cultured to confluence in DMEM medium with supplement of $10 \%$ FBS under either simulated microgravity condition or static condition. Peripheral whole blood was collected from a healthy donor in Vacutainer cell tubes containing sodium citrate.

Peripheral blood mononuclear cells (PBMCs) were immediately separated by centrifugation, washed twice with PBS.

PBMCs at G0 phase and confluent M10 cells (mainly in G1 phase) were fixed in fresh Methanol: Acetic Acid (3:1) fixative. Fixed samples were then washed with $1 x$ PBS without air dry. The samples were immediately hybridized with the XCyte 3 mBAND kit (MetaSystems) which distinct the chromosome in 23 different color bands. Three-dimensional images of each of the six painted regions of chromosome3 were captured with a Zeiss and a Leica laser confocal microscope (Fig.1). The captured image was uploaded into Imaris $($ and Fuji image analysis software. The surface of each of the six chromosome regions was determined (Fig.1c). The center of each region and the center (not centrosome) of the entire chromosome domain were calculated once the surface is defined.

mBAND techniques

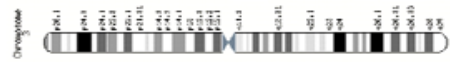

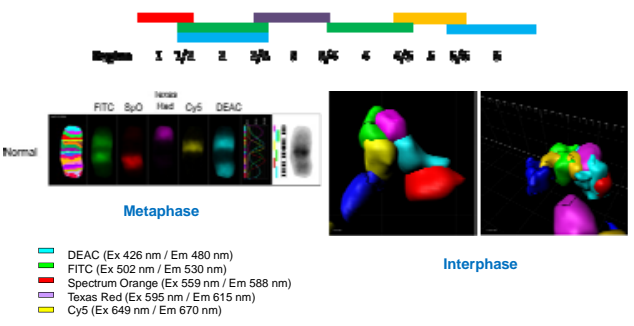

Figure 1. Examples of chromosome 3 of human lymphocytes painted 4 with $\mathrm{mBAND}$ (A and B) and chromosome 3 and 6 with FISH (C) after red arrow (grey arrows indicating normal chromosomes) include translocations, insertion, and deletion.

\section{RESULTS}

1. Distances between the center of each region and the center of the chromosome domain

The telomere regions (Regions 1 and 6), as well as the q-arm of the chromosome (Regions 4-6) appeared to be located towards the exterior of the chromosome domain. Most of the regions in the p-arm of the chromosome (Regions $1 / 2-3$ ), as well as the centromere region (Region 3/4), however, were closer to the center of the chromosome domain.

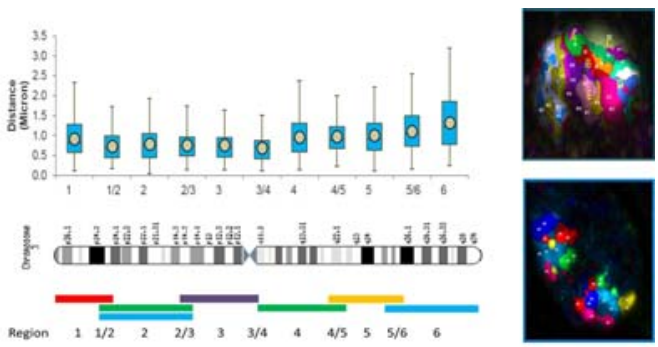

Figure 2. Distances between the center of each region and the center of the chromosome domain. The bars represent minimum and maximum of the measured value, and the circle the average value. The shaded box represents the 25th to75th percentile and the horizontal line the 50th percentile of the measured value.

2. Distances between the center of each region and the center of the chromosome domain

Within the p-arm of the chromosome, the physical distance increased as the genomic distance increased. However, the distance between Region2 and other regions on the q-arm of the chromosome appeared to be weakly dependent on the genomic separation.

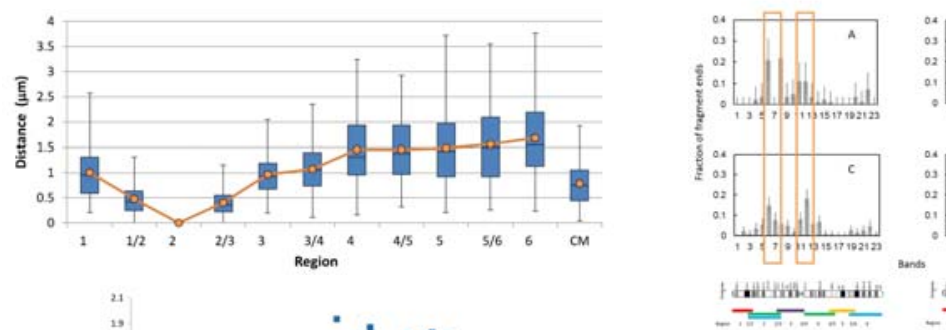
regions as a function of the genomic separation. The round dots indicate the distance between two regions on the same arm of the chromosome, whereas, chromosome.

3. The angles extended from the center of each colored region to the adjacent colored regions

The mean angle is the smallest for the regions on the p-arm of the chromosome in comparison to the q-arm. However, the mean angle for the centromere region appeared to be greater than the angles for regions on either the p-or q-arm.

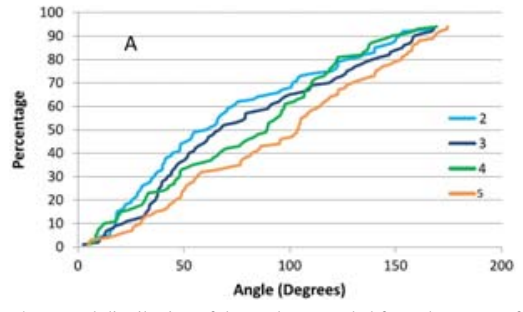

Figure 4. Integral distribution of the angles extended from the center of each colored region to the adjacent colored regions.
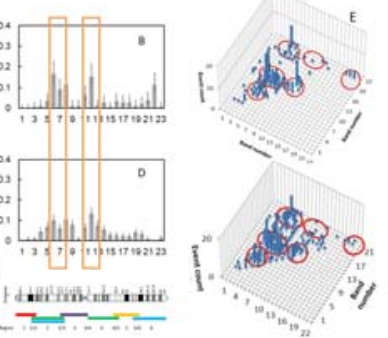

Figure 6. Intrachromosome exchanges occur frequently by rejoining of one break in band 6-8 (3p21) and one in band 11-12 (3q11) in M10 cells after highLET or low-LET radiation exposure. Hada et al. Rad Res., 2011

5. Factors that determine the $3 \mathrm{D}$ structure of interphase chromosome 3

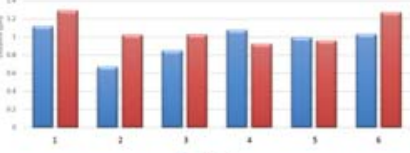

4. Region 2 in intrachromsomal aberrations

Aberrations involving chromosome 3 are associated with a number of known cancer types. Most of breaks involved in intra-chromosome 3 region (within region 2) is a known fragile site and contains several tumor pression genes.

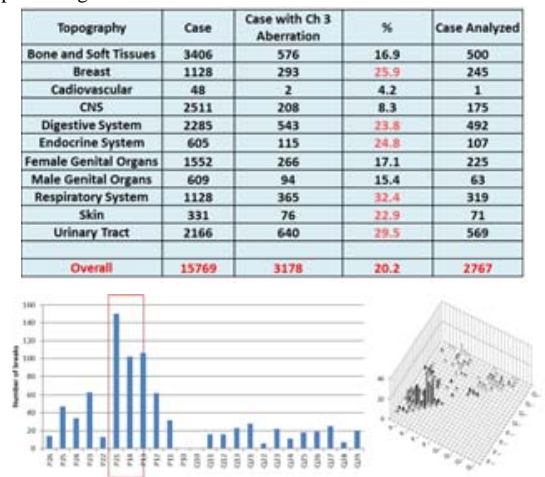

Figure 5. Distributions of intra-chromosome 3 exchanges in cancer patients. Mitelman Database of Chromosome Aberration and Gene Fusions in Cancer (2013). Mitelman F, Johansson B, and Mertens F (Ed.),

http://cgap.nci.nih.gov/Chromosomes/Mitelman

es between the center of each region and the center of the chromosome domain in M10 cells, and M10 cells grown under simulated microgravity.

\section{CONCLUSIONS}

- On a multi-megabase pair scale of the DNA, the arrangement of chromatin in M10 epithelial cells is non-random.

- In human epithelial cells, Both telomere regions tended to be located towards the exterior of the chromosome domain, whereas the centromere region towards the interior. In addition, the interior of the chromosome domain was preferentially occupied by the p-arm of the chromatin, which is consistent with our previous finding of intrachromosome exchanges involving breaks on the p-arm and in the centromere region of chromosome 3 .

- Chromosome conformation is cell type dependent, and is altered in a significant number of cells under various culture conditions, such as simulated microgravity, particularly in the regions close to mid-p arm (3p21), centromere, and 3' telomere.

Acknowledgement: We greatly appreciate the support from the NSRL team at Brookhaven National Laboratory and HIMAC facility. This work is supported by the NASA Space Radiation Health Program. 